\section{Respuesta Carta al Editor}

\section{Señor Editor:}

Tal como comenta el Dr. Cavagnaro en su carta al Editor titulada "Malformaciones nefro-urológicas en niños con síndrome de Down”, la frecuencia de morbilidad nefrourológica en niños con síndrome de Down se encuentra aumentada respecto a la población general, de acuerdo a los descrito por diversos autores ${ }^{1-3}$, sin embargo, a pesar de ello, los grupos de expertos aún no llegan a consenso sobre la costo-efectividad de realizar test de tamizaje con ecografía renal y vesical al nacer o evaluación de función renal periódica, pues los problemas nefrourológicos pueden ser benignos y transitorios como las pielectasias o pueden manifestarse en la evolución posterior del niño, como son la litiasis o el trastorno de vaciamiento vesical y por otro lado, los hallazgos funcionales, estar condicionados por mala hidratación.

En la experiencia de nuestro Programa de seguimiento en Salud de Personas con Síndrome de Down de la Red de Salud UC CHRISTUS, disponemos de datos aún no publicados, con información de 871 niños y adolescentes entre 0 y 20 años, donde encontramos una frecuencia de $9,5 \%$ de niños con problemas nefrourológicos $(\mathrm{n}=83)$. Al revisar el detalle de esas morbilidades, encontramos niños con más de una morbilidad, distribuyéndose de la siguiente forma: $9 \%$ de niños con infección urinaria $(\mathrm{n}=78), 5,8 \%$ de niños con pielectasia uni o bilateral ( $\mathrm{n}=51), 1,8 \%$ Hipospadia ( $\mathrm{n}=8$ de 440 hombres); $1,1 \%$ de Hidroureteronefrosis, $0,8 \%$ litiasi, incluyendo nefrocalcinosis, $0,6 \%$ de niños con quistes renales y la misma frecuencia de trastornos del vaciamiento vesical $(n=5), 0,44 \%$ con riñón ectópico o riñón en herradura, $0,3 \%$ de niños con reflujo vesicoureteral y la misma frecuencia para displasia renal $(\mathrm{n}=3)$. De los niños con Infección urinaria, 14 de 78 (18\%) tenían ecografía renal y vesical alterada, encontrándose 9 niños con pielectasia, 3 con hidroureteronefrosis sin reflujo vesicoureteral, un niño con reflujo vesicoureteral y otro con quistes renales.

La experiencia de nuestro Centro refleja la alta frecuencia de problemas nefrourológicos comentados por el Dr. Cavagnaro y otros autores ${ }^{1-3}$; sin embargo, pareciera ser necesario realizar estudios de costo efectividad en el uso de ecografía renal y vesical como tamizaje para detección de malformaciones nefrourológicas en el periodo de recién nacido, como para recomendar dicho estudio a todos los recién nacidos con síndrome de Down.

Mientras esa recomendación no sea respaldada por estudios, sería recomendable tomar cada instancia po- sible para evaluar la condición renal del niño o niña, en especial en aquellos con mayor riesgo.

Esta evaluación puede comenzar en aquellos con diagnóstico prenatal, buscando activamente la presencia malformaciones nefrourológicas y dilataciones de las vías urinarias en el feto con síndrome de Down, de manera de obtener un grupo seleccionado para confirmación en etapa postnatal. Si bien en Chile, el diagnóstico prenatal es bajo, alcanzando un $30 \%$ en el grupo de pacientes en seguimiento en nuestro Centro, es una instancia que no se debe perder para tener una mejor evaluación del futuro recién nacido.

Adicionalmente, sería recomendable realizar estudio completo y estricto con ecografía renal y vesical a aquellos con infección urinaria febril y no febril, a aquellos con uso prolongado de furosemida, especialmente aquellos con cardiopatía congénita hemodinámicamente significativa, aquellos con morbilidad grave que han requerido uso de furosemida por otras causas y en aquellos con dificultad en el control de esfínter o retencionistas, quienes pudieran tener trastornos en el vaciamiento vesical, además de aquellos con antecedentes familiares de algún problema renal eventualmente heredable.

Considerando que la ecografía renal y vesical es un buen test diagnóstico de malformaciones nefrourológicas, que no tiene efectos adversos para el niño, pareciera ser la mejor herramienta para aproximarse al diagnóstico, sin embargo, se debe también tener en cuenta que es un test operador dependiente, por lo que si se decide estudiar, sería recomendable realizar en un centro donde se cuente con profesionales entrenados en ecografía de niños.

Al evaluar la necesidad de estudios de función renal en este grupo, una buena propuesta sería agregarlo a los exámenes de rutina que ellos requieren anualmente, aprovechando el mismo momento de toma de muestra, sin embargo, pareciera relevante considerar algunos puntos a la hora de interpretar los resultados: 1) Sería importante conocer la función renal en aquellos con mayor riesgo, tales como pacientes con cardiopatías congénitas hemodinámicamente significativas y que requerirán de cardiocirugía; antecedentes de malformaciones nefrourológicas; usuarios de medicamentos nefrotóxicos o de uso de medios de contraste endovenoso; antecedentes familiares de riesgo para desarrollar nefropatías, entre otras; 2) Conocer las diferencias en el metabolismo de uratos descritas en este grupo, considerando que frente a niveles de urea elevada no se ha descrito asociación con gota más que en reportes de casos aislados ${ }^{4-6}$, incluso en uno de esos reportes refieren que la condición de síndrome de Down suprimiría la aparición de artritis por esta causa, ya que los niveles de uricemia necesarias para desarrollar artritis son mayores que las perso- 
nas con gota sin síndrome de Down 6 ; 3) Considerar la alta frecuencia de la dificultad para tomar agua, lo que hemos observado en el gran grupo en seguimiento y descrito en literatura reciente 7 , por lo que es muy frecuente encontrar con nitrógeno ureico plasmático sutilmente elevado frente a situaciones de poca hidratación o por las horas de ayuno necesario para la toma de exámenes. En la experiencia de nuestro Centro nos hemos encontrado con un grupo importante de niños estudiados en forma exhaustiva por nitrógeno ureico levemente elevado, que simplemente estaban en un contexto de deshidratación. Frente a esto último, la intervención más efectiva pareciera ser la recomendación de hidratación generosa diaria y evaluación clínica de hidratación, evitando estados crónicos de deshidratación leve, en especial en aquellos que viven en lugares expuestos a altas temperaturas, en temporadas estivales, en aquellos que viven en altura, en intercurrencias de infecciones respiratorias y digestivas y en aquellos con trastorno de deglución a líquidos claros, quienes son los que con mayor frecuencia rechazan tomar agua; 4) Tener en cuenta la dificultad que se enfrenta el equipo de enfermería para tomar la muestra, especialmente a la hora de interpretar los resultados de electrolitos plasmáticos (solicitados para evaluar funcionalidad renal), donde frente a una toma de muestra dificultosa se puede desencadenar hemólisis y elevación subsecuente de potasio, sin necesariamente tener hiperkalemia real.

En resumen, y en respuesta a lo comentado y propuesto por el Dr. Cavagnaro, es importante considerar las malformaciones nefrourológicas como un problema de salud de alta frecuencia en niños con síndrome de Down, por lo que sería recomendable fomentar estudios que evalúen la costo-efectividad de realizar un test de tamizaje en recién nacidos. Mientras tanto, si es que hay recursos disponibles, la ecografía renal en el periodo de recién nacido pudiera ser una adecuada conducta, sin embargo, si ésta no está disponible, sería recomendable extremar las medidas de alta sospecha de malformaciones, considerando otras instancias en las que se puedan evaluar riñones y vejiga, y educar en hidratación generosa preventiva y evitar morbilidad renal, especialmente al decidir usar medicamentos nefrotóxicos y durante intercurrencias.

\section{Referencias}

1. Kupferman J, Druschel C, Kupchnik S. Increased prevalence of renal and urinary tract anomalies in children with Down syndrome. Pediatrics. 2009;124: e615-21.

2. Málaga S, Pardo R, Málaga I, Orejas G, FernándezToral J. Renal involvement in Down syndrome. Pediatr Nephrol. 2005;20(5):614-7.

3. Jain M, Singh A, Mantan M, Kapoo S. Evaluation of Structural Anomalies of Kidney and Urinary Tract in Children with Down Syndrome. The Indian Journal of Pediatrics 2014;81:734

4. Nishida Y, Akaoka I, Kobayashi M, Maruki K, Oshima Y. Renal impairment in urate excretion in patients with Down's syndrome. J Rheumatol. 1979;6(1):103-7.

5. Ciompi ML, Bazzichi LM, Bertolucci D, et al. Uric acid metabolism in two patients with coexistent Down's syndrome and gout. Clin Rheumatol. 1984;3(2):229-33.

6. Kamatani N, Yamanaka H, Totokawa S, Kashiwazaki S, Higurashi M. Down syndrome with coexistent gout: report of six patients and possible reasons for the scarcity of descriptions of this association. Ann Rheum Dis. 1996;55(9):649-50.

7. Magenis ML, Machado AG, Bongiolo AM, da Silva MA, Castro K, Perry ID. Dietary practices of children and adolescents with Down syndrome. J Intellect Disabil. Epub: 2017 Jan 1:1744629516686571. doi: $10.1177 / 1744629516686571$.

Macarena Lizama C.

Pediatra, Profesor Asistente, Facultad de Medicina, Pontificia Universidad Católica de Chile. Centro UC Síndrome de Down. 